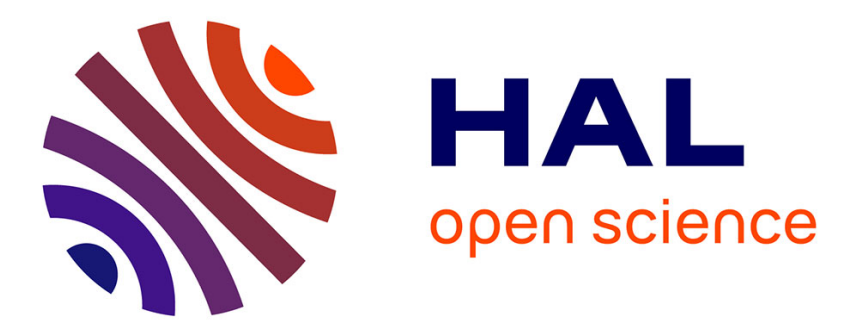

\title{
Theory of propagation of elastic waves in a fluid-saturated porous solid. II. Higher frequency range
}

\author{
Maurice A. Biot
}

\section{To cite this version:}

Maurice A. Biot. Theory of propagation of elastic waves in a fluid-saturated porous solid. II. Higher frequency range. Journal of the Acoustical Society of America, 1956, 28 (2), pp.179-191. 10.1121/1.1908241 . hal-01368668

\section{HAL Id: hal-01368668 https://hal.science/hal-01368668}

Submitted on 21 Sep 2016

HAL is a multi-disciplinary open access archive for the deposit and dissemination of scientific research documents, whether they are published or not. The documents may come from teaching and research institutions in France or abroad, or from public or private research centers.
L'archive ouverte pluridisciplinaire HAL, est destinée au dépôt et à la diffusion de documents scientifiques de niveau recherche, publiés ou non, émanant des établissements d'enseignement et de recherche français ou étrangers, des laboratoires publics ou privés. 


\title{
Theory of Propagation of Elastic Waves in a Fluid-Saturated Porous Solid. II. Higher Frequency Range
}

\author{
M. A. Brot* \\ Shell Development Company, RCA Building, New York, New York
}

(Received September 1, 1955)

\begin{abstract}
The theory of propagation of stress waves in a porous elastic solid developed in Part I for the low-frequency range is extended to higher frequencies. The breakdown of Poiseuille flow beyond the critical frequency is discussed for pores of flat and circular shapes. As in Part I the emphasis of the treatment is on cases where fluid and solids are of comparable densities. Dispersion curves for phase and group velocities along with attenuation factors are plotted versus frequency for the rotational and the two dilational waves and for six numerical combinations of the characteristic parameters of the porous systems. Asymptotic behavior at high frequency is also discussed.
\end{abstract}

\section{INTRODUCTION}

A PREVIOUS paper ${ }^{1}$ dealing with the subject of propagation of elastic waves in a fluid saturated porous solid was restricted to the low-frequency range. By this it was meant a frequency range between zero and a certain value beyond which the assumption of Poiseuille flow broke down. The purpose of this paper is to extend the theory to the full frequency range without the limitation of the foregoing assumption. There remains however an upper bound for the frequency, namely, that at which the wavelength becomes of the order of the pore size. Such a case must, of course, be treated by a different method.

A theoretical study of the breakdown of Poiseuille flow is presented in Secs. 2 and 3, by considering the flow of a viscous fluid under an oscillatory pressure gradient either between parallel walls or in a circular tube. The case of the circular tube was originally treated by Kirkhoff. This study yields a complex viscosity correction factor function of the frequency through the dimensionless ratio $f / f_{c}$ where $f_{c}$ is a characteristic frequency of the material. The case of flow between parallel walls and that of the circular tube indicate that the effect of pore cross-sectional shape is well represented by taking the same function of the frequency for the viscosity correction and simply changing the frequency scale. As in Part I we are primarily concerned with applications to liquids and we have neglected the thermoelastic effects.

Application of these results to fluid friction in a porous material is discussed in Sec. 4 and a "structural factor" is introduced which represents the effect of sinuosity and shape of the pores.

The propagation of rotational waves is discussed in Sec. 5. Four numerical combinations of parameters are considered. Group velocity, phase velocity, and attenuation are plotted for these four cases as a function of the frequency ratio $f / f_{c}$. There is only one type of rotational wave. The influence of the structural factor is also

\footnotetext{
* Consultant.

* Consultant.
1 M. A. Biot, J. Acoust. Soc. Am. 28, 168 (1956), preceding paper.
}

evaluated by calculating phase velocity and attenuation for a typical case.

The propagation of dilatational waves is discussed in Sec. 6. Group velocity, phase velocity, and attenuation curves are plotted for six numerical combinations of the parameters. There are two types of such waves, designated as waves of the first and second kind. The latter are characterized by high attenuation. An interesting plot is that of the attenuation per cycle. Both the rotational waves and the waves of the first kind exhibit a maximum value of this attenuation in a range of $f / f_{c}$ near unity. In this range the inertia and viscous forces are of the same order.

As discussed in Part I when the dynamic compatibility condition is satisfied or nearly satisfied $\left(z_{1} \cong 1\right)$ the wave of the first kind has a very small attenuation. This is shown by cases 2 and 5 . The other two waves, however, retain much higher attenuation. In such a case only one type of wave may be observed unless special attention is given to the others. Another aspect of this phenomenon will be exhibited when a dilatational wave is reflected at a surface of discontinuity. The reflected energy is split up into three types of waves, two of which may be unobserved because of their high attenuation. The phenomenon then appears as the propagation of a single-type body wave with small attenuation in the body and a high absorption at the reflection surface.

Certain assumptions upon which the present theory is based, such as perfect elasticity of the solid, limitations on the nonuniformity of pore size, and the neglection of thermal effects will determine the categories of materials and frequency ranges for which it is applicable. It should, however, be of value beyond its strict applicability by indicating orders of magnitudes or qualitative trends.

In applications to wave propagation in such materials as clay, silts, or muds, one should note that the rotational wave is determined entirely by the shearing rigidity of the solid. Since the latter may be small, the rotational waves may, in this case, propagate with a velocity which is considerably lower than that of the dilatational waves of first and second kind. 


\section{OSCILLATORY FRICTION FORCE IN A TWO-DIMENSIONAL DUCT}

We are interested in the motion of a fluid in a twodimensional duct, i.e., a space limited by two parallelplane boundaries when these boundaries are subject to an oscillatory motion and when an oscillatory pressure gradient acts at the same time on the fluid.

We consider only the two-dimensional motion and neglect all pressure gradients and velocity components normal to the boundaries. The $x$-direction is parallel to the boundaries and the $y$-axis is normal to it with the boundaries represented by $y= \pm a$. The $x$-component of the velocity of the boundary is $\dot{u}$ and that of the fluid $\dot{U}$ (Fig. 1). The latter component has a distribution along $y$ which is to be determined.

The equation of motion of the fluid in the $x$-direction is

$$
\rho_{f} \ddot{U}=-\frac{\partial p}{\partial x}+\mu \frac{\partial^{2} \dot{U}}{\partial y^{2}},
$$

where $\mu$ is the viscosity, $\rho_{f}$ the mass density of the fluid, and $p$ the pressure. Introducing the relative velocity of the fluid,

we write,

$$
U_{1}=\dot{U}-\dot{u}
$$

We may consider

$$
\rho_{f} \dot{U}_{1}=-\frac{\partial p}{\partial x}-\rho_{f} \ddot{u}+\mu \frac{\partial^{2} U_{1}}{\partial y^{2}} .
$$

$$
X \rho_{f}=-\frac{\partial p}{\partial x}-\rho_{f} \ddot{u}
$$

to be equivalent to an external volume force and Eq. (2.3) becomes

$$
\begin{gathered}
\dot{U}_{1}=X+\nu \frac{\partial^{2} U_{1}}{\partial y^{2}} \\
\nu=\mu / \rho_{f} .
\end{gathered}
$$

Assuming that all quantities are sinusoidal functions of time with a factor $e^{i \alpha t}$, and rewriting Eq. (2.5) without this factor we have

$$
\frac{d^{2} U_{1}}{d y^{2}}-i \alpha U_{1}=-X .
$$

The general solution to this equation is

$$
U_{1}=\frac{X}{i \alpha}+C \cosh \left[\left(\frac{i \alpha}{\nu}\right)^{\frac{3}{2}} y\right]
$$

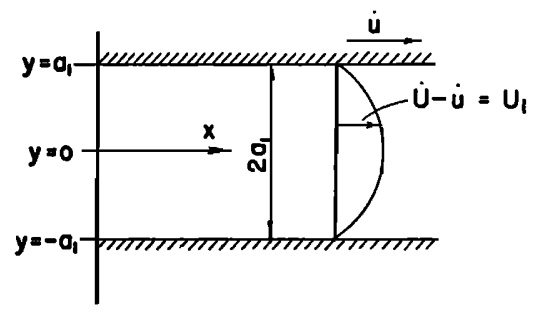

Fig. 1. Two-dimensional flow between parallel walls. with the condition that the function be symmetric in $y$. The constant $C$ is determined by the condition $U_{1}=0$ at the wall, i.e., for $y= \pm a_{1}$

$$
C=-\frac{X}{i \alpha} \frac{1}{\cosh \left[\left(\frac{i \alpha}{\nu}\right)^{\frac{1}{2}} a_{1}\right]} .
$$

The velocity distribution is

$$
U_{1}=\frac{X}{i \alpha}\left\{1-\frac{\cosh \left[(i \alpha / \nu)^{\frac{1}{3}} y\right]}{\cosh \left[(i \alpha / \nu)^{\frac{1}{2}} a_{1}\right]}\right\} .
$$

For use in the general theory, we shall need both the average velocity of the fluid through the cross section and the friction force at the wall. The average velocity $U_{1 \text { (Av) }}$ is given by

$$
2 a_{1} U_{1(\mathrm{Av})}=\frac{X}{i \alpha} \int_{-a_{1}}^{+a_{1}}\left\{1-\frac{\cosh \left[(i \alpha / \nu)^{\frac{1}{2}} y\right]}{\cosh \left[(i \alpha / \nu)^{\frac{1}{2}} a_{1}\right]}\right\} d y .
$$

Hence,

$$
U_{1 \text { (Av) }}=\frac{X}{i \alpha}\left\{1-\frac{1}{a_{1}}\left(\frac{\nu}{i \alpha}\right)^{\frac{1}{2}} \tanh \left[\left(\frac{i \alpha}{\nu}\right)^{\frac{1}{2}} a_{1}\right]\right\} .
$$

The friction stress at the wall $\left(y=-a_{1}\right)$ is

$$
\tau=\mu\left[\frac{d U_{1}}{d y}\right]_{y_{=-a_{1}}}=\frac{X \mu}{i \alpha}\left(\frac{i \alpha}{\nu}\right)^{\frac{1}{2}} \tanh \left[\left(\frac{i \alpha}{\nu}\right)^{\frac{1}{2}} a_{1}\right] .
$$

In applying these results we need the expression for the total friction force $2 \tau$ excited by the fluid on the wall, per unit average velocity of the fluid relative to the wall, i.e., we must calculate the ratio

$$
\frac{2 \tau}{U_{1(\mathrm{Av})}}=\frac{2 \mu}{a_{1}} \frac{a_{1}\left(\frac{i \alpha}{\nu}\right)^{\frac{1}{2}} \tanh \left[\left(\frac{i \alpha}{\nu}\right)^{\frac{1}{2}} a_{1}\right]}{1-\frac{1}{a_{1}}\left(\frac{\nu}{i \alpha}\right)^{\frac{1}{2}} \tanh \left[\left(\frac{i \alpha}{\nu}\right)^{\frac{1}{2}} a_{1}\right]} .
$$

In this expression we have a nondimensional variable.

and we write

$$
\kappa_{1}=a_{1}(\alpha / \nu)^{\frac{2}{2}},
$$

$$
\frac{2 \tau}{U_{1 \text { (AV) }}}=\frac{2 \mu}{a_{1}} \frac{i^{\frac{1}{2}} \kappa_{1} \tanh \left(i^{\frac{1}{\kappa}} \kappa_{1}\right)}{1-\frac{1}{i^{\frac{1}{2}} \kappa} \tanh \left(i^{\frac{1}{2}} \kappa_{1}\right)}
$$

Let us examine the limiting case when the frequency tends to zero, i.e., for $\kappa_{1} \rightarrow 0$. We have

$$
2 \tau / U_{1(\text { Av) }}=6 \mu / a_{1} \text {. }
$$


This corresponds to Poiseuille flow. In this case the velocity profile for $U_{1}$ if parabolic. If we put

$$
F_{1}(\kappa)=\frac{1}{3} \frac{i^{\frac{1}{}} \kappa_{1} \tanh \left(i^{1} \kappa_{1}\right)}{1-\frac{1}{\kappa_{1} i} \tanh \left(i^{\frac{1}{1}} \kappa_{1}\right)}
$$

we have $F_{1}(0)=1$, and we write

$$
\frac{2 \tau}{U_{1 \text { (Av) }}}=\frac{6 \mu}{a_{1}} F_{1}\left(\kappa_{1}\right),
$$

where $F_{1}\left(\kappa_{1}\right)$ is a complex quantity which is equal to unity for $\kappa_{1}=0$ and represents the deviation from Poiseuille friction as the frequency increases. There is a difference of phase between the velocity and the friction force. For large values of the frequency, i.e., $\kappa_{1} \rightarrow \infty$,

Fig. 2. Frequency correction functions for the viscosity in two-dimensional flow.

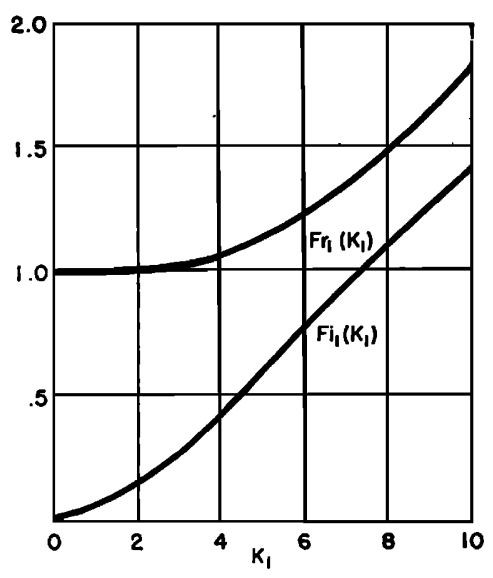

we have

$$
F_{1}\left(\kappa_{1}\right)=\frac{\kappa_{1}}{3} i=\frac{\kappa_{1}}{3}\left(\frac{1+i}{\sqrt{2}}\right),
$$

therefore, the friction force at large frequencies and for constant velocity increases like the square root of the frequency and is 45 degrees out of phase with the velocity. Everything happens as if the static viscosity coefficient $\mu$ were replaced by a dynamic value.

$$
\mu F_{1}\left(\kappa_{1}\right) \text {. }
$$

We separate the real and imaginary part of $F_{1}\left(\kappa_{1}\right)$ as follows:

$$
F_{1}\left(\kappa_{1}\right)=F r_{1}\left(\kappa_{1}\right)+F i_{1}\left(\kappa_{1}\right) .
$$

The values of $F r_{1}\left(\kappa_{1}\right)$ and $F i_{1}\left(\kappa_{1}\right)$ are plotted in Fig. 2 . It is seen that in accordance with Eq. (2.19) these curves become asymptotically parallel to straight lines of slope $\kappa_{1} / 3 \sqrt{2}=0.234 \kappa_{1}$ for large values of $\kappa_{1}$.

\section{OSCILLATORY FRICTION FORCE IN A THREE-DIMENSIONAL DUCT}

We shall now solve the same problem as in Sec. 2 except that instead of a two-dimensional motion be-

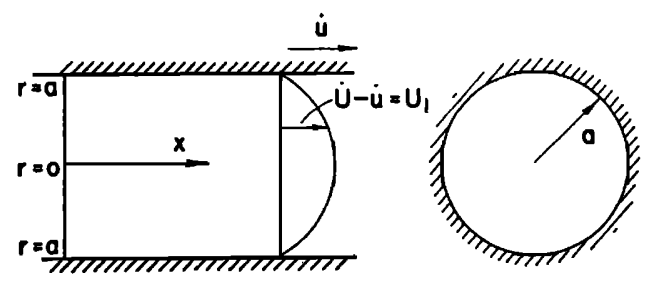

Fig. 3. Three-dimensional flow in a circular duct.

tween two plane boundaries we now consider a straight duct of circular cross section, (Fig. 3) of radius $a$. As in the foregoing, we consider the components of the motion and the pressure gradient along the direction $x$ of the axis. In this case Eq. (2.1) is replaced by

$$
\rho_{f} \ddot{U}=-\frac{\partial p}{\partial x}+\mu \nabla^{2} \dot{U}
$$

where $\nabla^{2}$ is the Laplacian operator. We assume that $\dot{U}$ is independent of $x$, and that the flow is axially symmetric so that the operator is

\section{Putting}

$$
\nabla^{2}=\frac{\partial^{2}}{\partial r^{2}}+\frac{1}{r} \frac{\partial}{\partial r}
$$

$$
-\frac{\partial p}{\partial x}-\rho_{f} \ddot{u}=X_{\rho_{f}}
$$

as before, and introducing the relative velocity $U_{1}$ $=\dot{U}-\dot{u}$ of the fluid with respect to the wall, we may write for Eq. (3.1)

$$
\nu\left(\frac{\partial^{2} U_{1}}{\partial r^{2}}+\frac{1}{r} \frac{\partial U_{1}}{\partial r}\right)-\frac{\partial U_{1}}{\partial t}=-X .
$$

All quantities being sinusoidal functions of time contain a factor $e^{i \alpha t}$. By rewriting Eq. (3.4) without this factor, we find

$$
\frac{d U_{1}}{d r^{2}}+\frac{1}{r} \frac{d U_{1}}{d r}-\frac{i \alpha}{\nu} U_{1}=-\frac{X}{\nu}
$$

This is a Bessel's differential equation for $U_{1}$. The general solution of this equation, which is finite at $r=0$, is

$$
U_{1}=\frac{X}{i \alpha}+C J_{0}\left[i\left(\frac{i \alpha}{\nu}\right)^{\frac{1}{2}} r\right],
$$

where $C$ is a constant and

$$
J_{0}(i \sqrt{ } i z)=b e r z+i b e i z
$$

with Kelvin functions of the first kind and zero order. Introducing the boundary condition $U_{1}=0$ for $r=a$

$$
\frac{i \alpha U_{1}}{X}=1-\frac{J_{0}\left[i\left(\frac{i \alpha}{\nu}\right)^{\frac{1}{2}} r\right]}{J_{0}\left[i\left(\frac{i \alpha}{\nu}\right)^{\frac{1}{2}} a\right]} .
$$


Again, here we need the average velocity for the cross section. The average velocity $U_{1(\mathrm{Av})}$ is given by

or

$$
U_{1(\mathrm{Av})}=\frac{2}{a^{2}} \int_{0}^{a} U_{1} r d r
$$

r

$$
\frac{i \alpha U_{1(\mathrm{Av})}}{X}=1-\frac{2}{k^{2} J_{0}\left(i^{\frac{3}{2}} k\right)} \int_{0}^{k} J_{0}\left(i^{\frac{1}{2}} \xi\right) \xi d \xi
$$

with

$$
\kappa=a\left(\frac{\alpha}{\nu}\right)^{\frac{2}{2}}
$$

The value of the integral in expression (3.10) is known. We have ${ }^{2}$

Hence,

$$
\int_{0}^{k} J_{0}\left(i^{\frac{3}{2}} \xi\right) \xi d \xi=\kappa\left(b e i^{\prime} \kappa-i b e r^{\prime} \kappa\right) .
$$

$$
\frac{i \alpha U_{1(\mathrm{Av})}}{X}=1-\frac{2}{i_{\kappa}} \frac{b e r^{\prime} \kappa+i b e i^{\prime} \kappa}{b e r \kappa+i b e i \kappa}
$$

In these expressions

$$
\begin{aligned}
& b e r^{\prime} z=\frac{d}{d z} b e r z \\
& b e i^{\prime} z=\frac{d}{d z} b e i z
\end{aligned}
$$

We also evaluate the friction between the fluid and the wall. The stress $r$ at the wall is

$$
\tau=-\mu\left[\frac{d U_{1}}{d r}\right]_{r=0}=\frac{X \mu}{i \alpha}\left(\begin{array}{c}
\alpha \\
-
\end{array}\right)^{\frac{1}{2}} .
$$

The total friction force is $2 \pi a \tau$, and the ratio of this force to the average velocity is

with

$$
\frac{2 \pi a \tau}{U_{1(\mathrm{Av})}}=\frac{2 \pi \mu \kappa T(\kappa)}{1-\frac{2}{i \kappa} T(\kappa)}
$$

$$
T(\kappa)=\frac{b^{\prime}{ }^{\prime} \kappa+i b e r^{\prime} \kappa}{\text { ber }^{\prime}+i b e i \kappa} .
$$

This formula is anologous to Eq. (2.15) where $T(\kappa)$ plays the role of $\sqrt{ } i \tanh \left(\sqrt{ } i_{\kappa_{1}}\right)$. We now consider the limiting value of expression (3.13) for $\kappa \rightarrow 0$, i.e., for very low frequency. We have

$$
\begin{aligned}
b e r \kappa+i b e i \kappa & =J_{0}(i \sqrt{ } i \kappa)=1+\frac{i \kappa^{2}}{2^{2}}-\frac{1}{2^{2} 4^{2}} \kappa^{4}+\cdots \\
b e r^{\prime} \kappa+i b e i^{\prime} \kappa & =\frac{i \kappa}{2}-\frac{\kappa^{3}}{16}+\cdots .
\end{aligned}
$$

${ }^{2}$ N. W. McLachlan, Bessel functions for Engineers (Clarendon Press, Oxford, England, 1934), p. 125.
Hence, in the limiting case $\kappa \rightarrow 0$,

and

$$
1-\frac{2}{i \kappa} T(\kappa) \rightarrow \frac{i \kappa^{2}}{8}
$$

$$
\underset{U_{1(\text { Av })}}{\stackrel{2 \pi a \tau}{\longrightarrow}} 8 \pi \mu \text {. }
$$

This expression checks with that obtained from Poiseuille flow. Again here we introduce a function

$$
F(\kappa)=\frac{1}{4} \frac{\kappa T(\kappa)}{1-\frac{2}{i \kappa} T(\kappa)}
$$

such that $F(0)=1$, and write

$$
\frac{2 \pi a \tau}{U_{1(\mathrm{Av})}}=8 \pi \mu F(\kappa) .
$$

The function $F(\kappa)$ measures the deviation from Poiseuille flow friction as a function of the frequency parameter $\kappa$. For large values of $\kappa$, i.e., at high frequency the asymptotic values are

$$
\begin{aligned}
& \text { ber } \kappa+i b e i \kappa \rightarrow \frac{1}{(2 \pi \kappa)^{\frac{1}{2}}} \exp \left[\kappa\left(\frac{1+i}{\sqrt{2}}\right)-\frac{i \pi}{8}\right] \\
& b e r^{\prime} \kappa+i b e i^{\prime} \kappa \rightarrow \frac{1}{(2 \pi \kappa)^{\frac{1}{2}}}\left(\frac{1+i}{\sqrt{2}}\right) \exp \left[\frac{\kappa(1+i)}{\sqrt{2}}-\frac{i \pi}{8}\right] .
\end{aligned}
$$

Hence,

$$
T(\kappa) \rightarrow \frac{1+i}{\sqrt{2}},
$$

and

$$
F(\kappa) \underset{4}{-}\left(\frac{1+i}{\sqrt{2}}\right)
$$

As in the two-dimensional case it is found that the friction at high frequency and for constant velocity is proportional to the square root of the frequency and is 45 degrees out of phase with the velocity. Everything happens as if the static viscosity coefficient $\mu$ were replaced by a dynamic complex value.

We put

$$
\mu F(\kappa) \text {. }
$$

$$
F(\kappa)=F r(\kappa)+i F i(\kappa) .
$$

The real and imaginary parts of this function are plotted in Fig. 4. For large value of $\kappa$ the curve becomes parallel to the straight lines. $\kappa / 4 \sqrt{2}=0.177 \kappa$.

\section{CALCULATION OF THE OSCILLATORY FRICTION FORCE IN A POROUS MATERIAL}

In applying the results of the previous section to a porous material, we introduce the assumption that the variation of friction with frequency follows the same laws as found in the foregoing for the tube of uniform 
cross section. It is also assumed that the pore size is fairly uniform. More will be said on this assumption later. We have considered two extreme cases, that of a duct limited by two planes and that of a circular duct. These cases correspond to extreme shapes in the cross section of the pores, i.e., whether they are close to very flat ellipses or to circles. In order to discuss this shape factor, let us consider the expression for the friction force as introduced in the previous paper. The friction force per unit volume of bulk material in the $x$-direction was expressed as

$$
b \frac{\partial}{\partial t}\left(U_{x}-u_{x}\right)
$$

This is the force exerted by the fluid on the solid in the direction of motion. The $x$-component of the average fluid velocity being $\partial U_{x} / \partial t$ and the velocity of the solid $\partial u_{x} / \partial t$. The quantity $\partial / \partial t\left(U_{x}-u_{x}\right)$ plays the same role as the relative average velocity $U_{1 \text { (Av) }}$ considered in the two previous sections. The coefficient $b$ is, therefore, the ratio of the total friction force to the average fluid velocity and may be expected to be multiplied by a frequency correction factor as in similar expressions (2.18) and (3.20) previously calculated. Hence, we write for the friction force

or

$$
b F_{1}\left(\kappa_{1}\right) \frac{\partial}{\partial t}\left(U_{x}-u_{x}\right)
$$

$$
b F(\kappa) \frac{\partial}{\partial t}\left(U_{x}-u_{x}\right)
$$

where $b$ is the coefficient for Poiseuille flow. Expression (4.2) corresponds to the case where the pore cross sections are more like narrow slits while Eq. (4.3) corresponds to the case where they are circular.

We shall now proceed to compare the two expressions (4.2) and (4.3) thereby evaluating the effect of pore shape on the frequency dependence of the friction. If we look at the plots of the functions $F_{1}\left(\kappa_{1}\right)$ and $F(\kappa)$ we notice that their shapes are very similar. A further check reveals that if we simply take $\kappa_{1}$ and $\kappa$ to be proportional, and if we take the scale such that the asymptotic directions become identical, i.e., by putting

$$
\frac{\kappa_{1}}{3 \sqrt{2}}=\frac{\kappa}{4 \sqrt{2}}
$$

the pair of curves $F r_{1}\left(\kappa_{1}\right) . F i_{1}\left(\kappa_{1}\right)$ become practically indistinguishable from $\operatorname{Fr}(\kappa), F i(\kappa)$ when the latter are plotted as function of $\kappa_{1}$. We may write with a good approximation

$$
\begin{aligned}
& F r_{1}\left(\kappa_{1}\right) \cong F r\left[(4 / 3) \kappa_{1}\right] \\
& F i_{1}\left(\kappa_{1}\right) \cong F i\left[(4 / 3) \kappa_{1}\right] .
\end{aligned}
$$

This means that when the pores have the shape of narrow slits its frequency dependence function may be
Fig. 4. Frequency correction functions for the viscosity in three-dimensional flow.

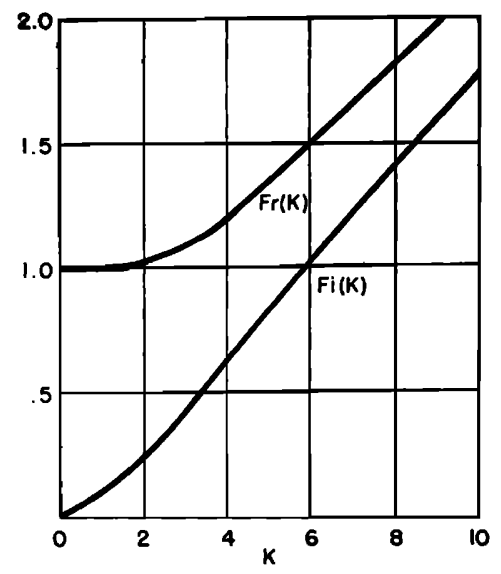

taken the same as for circular pores with a radius $a=4 / 3 a_{1}$.

This is also equivalent to saying that in the extreme case of slits and circular pores the effect of the frequency on the function is the same except for a change in scale of the frequency parameter. It is natural to assume that if this is true for the extreme case it is also true for intermediate shapes. A universal complex function $F(\kappa)$ may, therefore, be adopted to represent this frequency effect with a nondimensional parameter,

$$
\kappa=a\left(\frac{\alpha}{-\frac{1}{2}}\right)^{\frac{1}{\nu}},
$$

and where $a$ is a length which is characteristic of both the size of the pores and their geometry. In the case of circular pores $a$ is equal to the radius while in the case of slits of opening $2 a_{1}$, the characteristic size is as we have seen,

$$
a=(4 / 3) a_{1} .
$$

In the present treatment we shall assume that all pores have the same characteristic size $a$ or equivalently that the frequency correction is practically the same for all pores. This would naturally not be true in a material where pore size is distributed over a very wide range. A redistribution of friction would then occur between pores as the frequency varies with a corresponding redistribution in the velocity pattern. However, the assumption of a single characteristic pore size should be applicable to a wide variety of actual materials. The case of widely different pore sizes will have to be considered in a more general theory.

As regards the question of the choice of the size parameter $a$, the best way of course is to choose it in such a way that the dispersion and attentuation curves fit the experimental data.

In reference 1 the frequency $f$ appeared through a nondimensional parameter $f / f_{c}$. This introduces a characteristic frequency.

$$
f_{c}=\frac{b}{2 \pi \rho_{2}} \text {. }
$$


It now remains to relate the present parameter $\kappa$ with the nondimensional variable $f / f_{c}$. We notice first that $\kappa^{2}$ may be put in the same form,

$$
\kappa^{2}=\frac{f}{f_{c}^{\prime}}
$$

with a characteristic frequency

$$
f_{c}^{\prime}=\frac{\nu}{2 \pi a^{2}}
$$

The problem is, therefore, to compare $f_{c}{ }^{\prime}$ with $f_{c}$. Now $f_{c}$ is determined by the low-frequency or steady-state friction which is characterized by the parameter $b$. It will, therefore, depend not only on the pore characteristic "radius" $a$ but also on the sinuosity and shape of the pores. In order to evaluate $f_{c}$ we must, therefore, introduce some assumption regarding these geometric factors.

We first consider the case where the pores are parallel tubes of radius $a$ in the direction of flow. To evaluate $b$ we remember that it is the total friction force between the fluid and the solid per unit volume of bulk material and per unit average relative velocity in the steadystate flow, i.e., at zero frequency. From Eq. (3.18) the total friction at $\kappa=0$ per tube and per unit length is

$$
2 \pi a \tau=8 \pi \mu U_{1} .
$$

In order to obtain the value per unit cross section of the tube we divide this expression by $\pi a^{2}$

$$
\frac{2 \tau}{a}=\frac{8 \mu}{a^{2}} U_{1(\mathrm{AN})} .
$$

Since the fluid cross section occupies a fraction $\beta$ (porosity) of the cross section of the bulk material the friction per unit bulk volume is

$$
\frac{2 \tau \beta}{a}=\frac{8 \mu \beta}{a^{2}} U_{1 \text { (Av) }} .
$$

On the other hand,

$$
\beta \rho_{f}=\rho_{2},
$$

(the fluid mass density $=\rho_{f}$ ). Hence,

$$
f_{c}=\frac{4 \nu}{\pi} \frac{\nu}{a^{2}}
$$

Comparing with Eq. (4.10), we derive the relation between $f_{c}$ and $f_{c}{ }^{\prime}$

$$
f_{c}=8 f_{c}^{\prime},
$$

and with $f_{c}$ instead of $f_{c}^{\prime}$ the expression for $\kappa^{2}$ is

$$
\kappa^{2}=8 \frac{f}{f_{c}},
$$

and the frequency correction function may be written

$$
F(\kappa)=F\left[\left(\frac{8 f}{f_{c}}\right)^{\frac{1}{2}}\right] .
$$

If, instead of assuming the pores to be made out of parallel tubes of diameter $a$, we take into account their sinuosity, we must multiply the expression for $b$ by a factor $\xi$ which is greater than unity and takes this effect into account. Then, we write

$$
b=\frac{8 \mu \beta \xi}{a^{2}} .
$$

The characteristic frequency is then

$$
f_{c}=\frac{4 \nu \xi}{\pi a^{2}}
$$

in which case

$$
\begin{aligned}
& f_{c}=8 \xi f_{c}^{\prime} \\
& \kappa^{2}=8 \xi \frac{f}{f_{c}} .
\end{aligned}
$$

The frequency correction function is

$$
F(k)=F\left[\left(8 \xi \frac{f}{f_{c}}\right)^{\frac{3}{3}}\right] .
$$

If we consider slit-like pores instead of circular pores we go back to relation (2.16) which gives the total friction between the fluid and the solid at zero frequency. We derive the friction per unit area of the fluid by dividing Eq. (2.16) by $2 a_{1}$, and we find

$$
\frac{\tau}{a_{1}}=\frac{3 \mu}{a_{1}^{2}} U_{1 \text { (Av) }} .
$$

By an argument similar to the case of the circular tube, the value of $b$ is found to be

$$
b=\frac{3 \mu \beta}{a_{1}{ }^{2}} .
$$

If the pores are not parallel but sinuous, we must multiply the expression by a factor $\xi$ and write

$$
b=\frac{3 \mu \beta \xi}{a_{1}{ }^{2}} .
$$

Now, it was derived by Eq. (4.7), that the value of $a$ to be introduced in $\kappa$ was $a=4 / 3 a_{1}$, hence, for this case

$$
f_{c}=\frac{8}{3 \pi} \frac{\nu \xi}{a^{2}}
$$


Hence,

$$
f_{c}=\frac{16}{3} \xi f_{c}^{\prime}
$$

The value of $\kappa^{2}$ is

$$
\kappa^{2}=\frac{16 f f}{3} f_{c} .
$$

The frequency correction function is then

$$
F(\kappa)=F\left[\left(\frac{16 f}{3} \frac{f}{f_{c}}\right)^{\frac{1}{3}}\right] .
$$

We see, therefore, that various geometric factors such as sinuosity and cross-section shape enter into the function $F$ by a factor multiplying $f / f_{c}$. In general, we may write

$$
F(\kappa)=F\left[\delta\left(\frac{f}{f_{c}}\right)^{\frac{1}{3}}\right],
$$

where $\delta$ is a factor dependent on the geometry of the pores. With a sinuosity factor $\xi(\xi>1)$, the expression for $\delta$ varies from

for circular pores, to

$$
\delta=(8 \xi)^{\frac{1}{3}},
$$

$$
\delta=\left(\frac{16}{3} \xi\right)^{\frac{1}{2}}
$$

for slit-like pores. This factor $\delta$ is referred to hereafter as the "structural factor."

The best value of $\delta$ should be determined, of course, by the experimental data itself, i.e., it should furnish the best fit for dispersion and attenuation data.

In the following numerical work we shall, however, choose arbitrarily a value which is taken to represent an average. We put

$$
\delta=\sqrt{ } 8 .
$$

For a value of the sinuosity factor satisfying the inequality

$$
1<\xi<\frac{3}{2},
$$

the value $\delta=\sqrt{ } 8$ is between the value (4.31) and (4.32) for the circular and slit-like pores.

\section{PROPAGATION OF ROTATIONAL WAVES}

The rotational waves are governed by the same equations as in the low-frequency range provided the viscosity is replaced by its effective value function of the frequency. This amounts to replacing the resistance coefficient $b$ by $b F(\kappa)$. With this substitution, Eq. (7.2) of reference 1 for the rotational waves become

$$
\begin{aligned}
& \frac{\partial^{2}}{\partial t^{2}}\left(\rho_{11} \omega+\rho_{12} \boldsymbol{\Omega}\right)+b F(\kappa) \frac{\partial}{\partial t}(\boldsymbol{\omega}-\mathbf{\Omega})=N \nabla^{2} \boldsymbol{\omega} \\
& \frac{\partial^{2}}{\partial t^{2}}\left(\rho_{12} \omega+\rho_{22} \boldsymbol{\Omega}\right)-b F(\kappa) \frac{\partial}{\partial t}(\boldsymbol{\omega}-\boldsymbol{\Omega})=0,
\end{aligned}
$$

where $\rho_{11} \rho_{12} \rho_{22}$ are mass density parameters for the solid the fluid and their inertia coupling, and $\omega$ and $\Omega$ represent the rotations of solid and fluid. The rigidity of the solid is represented by the modulus $N$.

We consider a plane rotational wave

$$
\begin{aligned}
& \omega=C_{1} \exp [i(l x+\alpha t)] \\
& \Omega=C_{2} \exp [i(l x+\alpha t)] .
\end{aligned}
$$

Substitution of these expressions into Eq. (5.1) leads to a characteristic equation

$$
\frac{N l^{2}}{\rho a^{2}}=E_{r}-i E_{i}
$$

We have introduced the mass density of the bulk material as,

We put

$$
\rho=\rho_{11}+2 \rho_{12}+\rho_{22} \text {. }
$$

and hence, we have

$$
\begin{aligned}
& \gamma_{11}=\frac{\rho_{11}}{\rho} \\
& \gamma_{12}=\frac{\rho_{12}}{\rho} \\
& \gamma_{22}=\frac{\rho_{22}}{\rho},
\end{aligned}
$$

$$
\gamma_{11}+2 \gamma_{12}+\gamma_{22}=1 \text {. }
$$

The characteristic frequency is

$$
f_{c}=\frac{l}{2 \pi \rho_{2}}=\frac{b}{2 \pi \rho\left(\gamma_{12}+\gamma_{22}\right)} .
$$

Also,

$$
\begin{aligned}
& \epsilon_{1}=\left(\gamma_{12}+\gamma_{22}\right) \frac{f_{c}}{f} F_{r} \\
& \epsilon_{2}=\left(\gamma_{12}+\gamma_{22}\right) \frac{f_{c}}{f} F_{i} .
\end{aligned}
$$

Then the real and imaginary parts in expression (5.3) may be written

$$
\begin{aligned}
& E_{r}=\frac{\left(\gamma_{11} \gamma_{22}-\gamma_{12}\right)^{2}\left(\gamma_{22}+\epsilon_{2}\right)+\gamma_{22} \epsilon_{2}+\epsilon_{1}{ }^{2}+\epsilon_{2}{ }^{2}}{\left(\gamma_{22}+\epsilon_{2}\right)^{2}+\epsilon_{1}{ }^{2}} \\
& E_{i}=\frac{\epsilon_{1}\left(\gamma_{12}+\gamma_{22}\right)^{2}}{\left(\gamma_{22}+\epsilon_{2}\right)^{2}+\epsilon_{1}{ }^{2}} .
\end{aligned}
$$

In deriving these values use was made of the identity (5.6). The case of Poiseuille flow friction law considered 


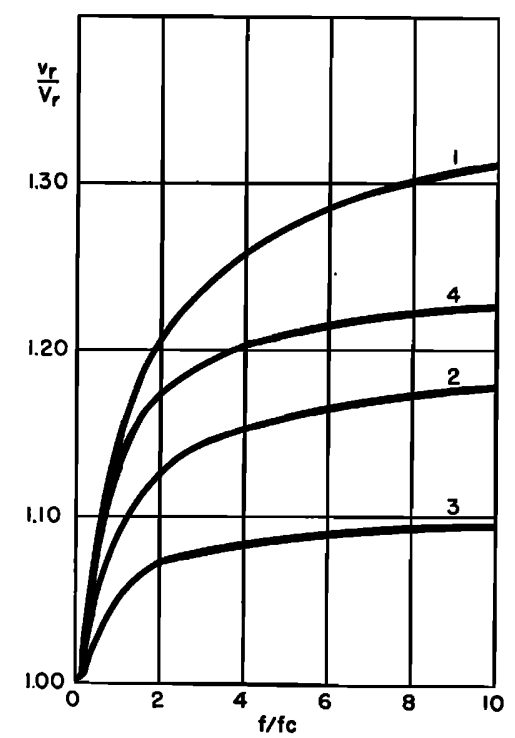

Fig. 5. Phase velocity $v_{r}$ of rotational waves. in reference 1 is found by putting

$$
\begin{aligned}
& \epsilon_{1}=\left(\gamma_{12}+\gamma_{22}\right) \frac{f_{c}}{f} \\
& \epsilon_{2}=0 .
\end{aligned}
$$

In order to derive the phase velocity and attenuation we proceed as before. We put

$$
l=l_{r}+i l_{i} .
$$

The phase velocity is then

$$
v_{r}=\frac{\alpha}{\left|l_{r}\right|} .
$$

We introduce the reference velocity

$$
V_{r}=\left(\frac{N}{\rho}\right)^{\frac{1}{2}}
$$

This is the velocity of rotational waves when the fluid and the solids are displaced together with no relative velocity. By using expression (5.3) we find

$$
\frac{v_{r}}{V_{r}}=\frac{\sqrt{2}}{\left[\left(E_{r}^{2}+E_{i}^{2}\right)^{\frac{1}{2}}+E_{r}\right]^{\frac{1}{2}}} .
$$

This is a function of $f / f_{c}$ with the parameters $\gamma_{i \gamma}$ and $\delta$. We have plotted these phase velocity dispersion curves using an average value $\delta=\sqrt{ } 8$ for the structural factor. Four cases have been considered for the dynamic parameter $\gamma_{i j}$ corresponding to cases marked one to four in Table $\mathrm{I}$.

The curves for $v_{r} / V_{2}$ have been plotted in Fig. 5 for these four cases as functions of $f / f_{c}$.
The behavior of the function for small value of the frequency was discussed in reference 1 . The limiting value of $v_{r} / V_{r}$ for $f \rightarrow 0$ is unity. At high frequency $\left(f / f_{c} \rightarrow \infty\right)$. The limiting values are

$$
F_{r}=F_{i}=\frac{\kappa}{4 \sqrt{2}}=\frac{1}{2}\left(\frac{f}{f_{c}}\right)^{\frac{1}{2}}
$$

$$
\begin{aligned}
& \epsilon_{1}=\epsilon_{2}=\frac{1}{2}\left(\gamma_{12}+\gamma_{22}\right)\left(\frac{f_{c}}{f}\right)^{\frac{3}{3}} \rightarrow 0 \\
& E_{r}=\left(\gamma_{11} \gamma_{22}-\gamma_{12}{ }^{2}\right) / \gamma_{22} \\
& E_{i}=\frac{1}{2} \frac{\left(\gamma_{12}+\gamma_{22}\right)^{3}}{\gamma_{22}{ }^{2}}\left(\frac{f_{c}}{f}\right)^{\frac{1}{2}} \rightarrow 0 .
\end{aligned}
$$

Hence, the limiting value of $v_{r} / V$ at high frequency is

$$
\frac{v_{r}}{V_{r}}=\left(\frac{\gamma_{22}}{\gamma_{11} \gamma_{22}-\gamma_{12}^{2}}\right)^{\frac{1}{2}}
$$

This may also be written

$$
v_{r}=\left[\frac{N}{\rho_{11}\left(1-\frac{\rho_{12}{ }^{2}}{\rho_{11} \rho_{22}}\right)}\right]^{\frac{1}{2}} .
$$

A value which coincides with Eq. (5.2) of reference 1 for the velocity in a porous medium containing a fluid with no viscosity. At large frequency we may neglect the influence of the viscosity on the velocity. All velocity dispersion curves for $v_{r} / V_{r}$ start at value unity for $f=0$ and tend toward the asymptotic value Eq. (5.22) for large frequencies.

In order to evaluate the attenuation of these waves, we introduce the length

$$
x_{a}=\frac{1}{\left|l_{i}\right|} .
$$

This length represents the distance through which the wave amplitude is attenuated by a factor $1 / e$. We also introduce a reference length

$$
L_{r}=\frac{V_{r}}{2 \pi f_{c}} .
$$

TABLE I.

\begin{tabular}{ccccccccc}
\hline \hline Case & $\sigma_{11}$ & $\sigma_{22}$ & $\sigma_{12}$ & $\gamma_{11}$ & $\gamma_{22}$ & $\gamma_{12}$ & $\mathrm{z}_{1}$ & $\mathrm{z}_{2}$ \\
\hline 1 & 0.610 & 0.305 & 0.043 & 0.500 & 0.500 & 0 & 0.812 & 1.674 \\
2 & 0.610 & 0.305 & 0.043 & 0.666 & 0.333 & 0 & 0.984 & 1.203 \\
3 & 0.610 & 0.305 & 0.043 & 0.800 & 0.200 & 0 & 0.650 & 1.339 \\
4 & 0.610 & 0.305 & 0.043 & 0.650 & 0.650 & -0.150 & 0.909 & 2.399 \\
5 & 0.500 & 0.500 & 0 & 0.500 & 0.500 & 0 & 1.000 & 1.000 \\
6 & 0.740 & 0.185 & 0.037 & 0.500 & 0.500 & 0 & 0.672 & 2.736 \\
\hline
\end{tabular}


We may then write

$$
\frac{L_{r}}{x_{a}}=\frac{f}{f_{c}} \frac{E_{i}}{\sqrt{2}}\left(E_{r}+\sqrt{ } E_{r}{ }^{2}+E_{i}{ }^{2}\right) .
$$

This expression $L_{r} / x_{a}$ is a nondimensional representation of the attenuation factor $\left|l_{i}\right|$ 'such that the wave amplitude as a function of the distance $x$ is proportional to $\exp \left[-\left|l_{i}\right| x\right]$. It is plotted as a function of $f / f_{c}$ in Fig. 6 for cases 1 to 4 of Table I. According to expression (7.17) in reference 1 the attenuation coefficient $L_{r} / x_{a}$ tends to zero like $\left(f / f_{c}\right)^{2}$. For large values of the frequency, using the asymptotic value Eqs. (5.20), (5.21) we find

$$
\frac{L_{r}}{x_{a}}=\frac{1}{4}\left(\frac{f}{f_{c}}\right)^{\frac{1}{2}} \frac{\left(\gamma_{12}+\gamma_{22}\right)^{2}}{\gamma_{22}{ }^{2}}\left(\frac{\gamma_{22}}{\gamma_{11} \gamma_{22}-\gamma_{12}{ }^{2}}\right)^{\frac{1}{2}} .
$$

The attenuation coefficient is proportional to $\sqrt{ } f$. It will be noted that the increase of the attenuation factor with frequency is due to the increase of the apparent viscosity with frequency, i.e., the fact that $F(\kappa)$ is not constant but becomes proportional to $\kappa$. If we had assumed the Poiseuille law to be valid at very high frequency, we would have found that the attentuation factors tend toward a constant value instead of increasing.

We shall also consider another quite significant quantity, namely, the attenuation coefficient per cycle instead of per unit distance. This quantity is $2 \pi\left|l_{i}\right| /$ $\left|l_{r}\right|$. Per cycle, the wave amplitudes is multiplied by

We find

$$
e^{-2 \pi\left|l_{i}\right| /\left|l_{r}\right|} \text {. }
$$

$$
2 \pi \frac{\left|l_{i}\right|}{\left|l_{r}\right|}=\frac{2 \pi E_{i}}{E_{r}+\left(E_{r}^{2}+E_{i}^{2}\right)^{\frac{1}{2}}} .
$$

This quantity is plotted in Fig. 7 for cases 1 to 4 of Table I. It is seen that the attenuation per cycle is zero at zero frequency, goes through a maximum, and tends to zero again at high frequency. The value near $f=0$ is

$$
2 \pi \frac{\left|l_{i}\right|}{\left|l_{r}\right|}=\pi \frac{f}{f_{c}}\left(\gamma_{12}+\gamma_{22}\right),
$$

Fig. 6. Attenuation coefficient of rotational waves.
Fig, 7. Attenuation per cycle of rotational waves.

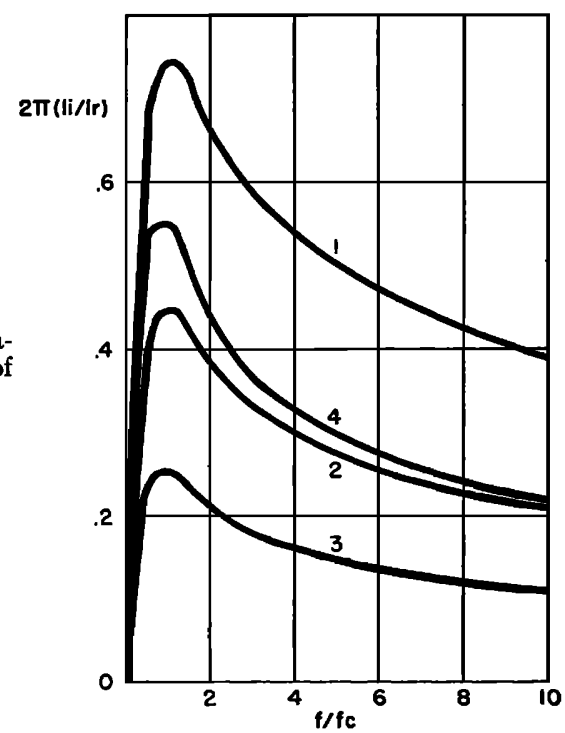

while for large $f$ it becomes

$$
2 \pi \frac{\left|l_{i}\right|}{\left|l_{r}\right|}=\frac{\pi}{2} \frac{\left(\gamma_{12}+\gamma_{22}\right)^{3}}{\gamma_{22}\left(\gamma_{11} \gamma_{22}-\gamma_{12}\right)^{2}}\left(\frac{f_{c}}{f}\right)^{\frac{1}{2}}
$$

It vanishes like $1 / \sqrt{ } f$. Finally, it remains to investigate the group velocity of the rotational waves. This group velocity is given by

Now,

$$
v_{g r}=\frac{d \alpha}{d\left|l_{r}\right|} .
$$

Hence,

$$
\frac{\alpha}{v_{r}}=\left|l_{r}\right| .
$$

$$
\frac{1}{v_{g r}}=\frac{d\left|l_{r}\right|}{d \alpha}=\frac{d}{d \alpha}\left(\frac{\alpha}{v_{r}}\right) .
$$

Introducing the reference velocity $V_{r}$, this may be written

$$
\frac{V_{r}}{v_{g r}}=\frac{d}{d\left(\frac{f}{f_{c}}\right)}\left[\frac{f}{f_{c}} \frac{V_{r}}{v_{r}}\right]
$$

The nondimensional group velocity variable $v_{g r} / V_{r}$ may be calculated from the foregoing dispersion curve $v_{r} / V_{r}$ by taking derivatives with respect to $f / f_{c}$. This was done analytically by using the formulas derived above for $v_{r} / V_{r}$. In so doing, it is noted that the derivative of $T(\kappa)$ [see Eq. (3.16)] which appears in these expressions may be written as follows if we take into account the properties of the Bessel functions.

With $J_{0}=J_{0}\left(i^{i} \kappa\right)$, we write

$$
T(k)=\frac{1}{J_{0}} \frac{d J_{0}}{d \kappa} .
$$




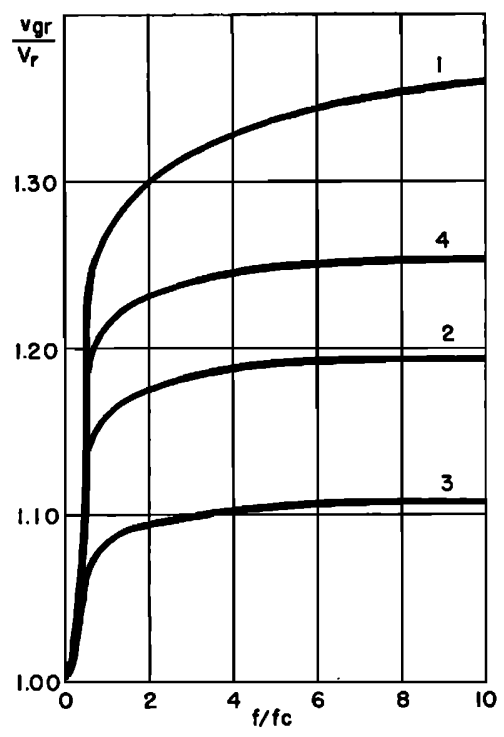

Since we have the identity

we derive

$$
\frac{d^{2} J_{0}}{d \kappa^{2}}+\frac{1}{\kappa} \frac{d J_{0}}{d \kappa}-i J_{0}=0,
$$

$$
\frac{d T}{d \kappa}=-T^{2}-\frac{1}{\kappa} T+i .
$$

The group velocity curves in the nondimensional form $v_{g r} / V_{r}$ have been plotted in Fig. 8 for cases 1 to 4 of Table I.

All foregoing cases have been computed for a value of the structural factor $\delta=\sqrt{ } 8$ considered to be an average value. In an extreme case of slit-like pores and a sinuosity factor $\xi=1$, we would have $\delta=(16 / 3)^{\frac{1}{2}}$ [see Eq. (4.32)]. In order to investigate the effect of the structural factor on the dispersion and attenuation of rotational waves, cases 1 to 4 of Table I were computed with the value $\delta=(16 / 3)^{\frac{1}{2}}$ and compared with the curves for $\delta=\sqrt{ } 8$. Velocity curves $v_{r} / V_{r}$ and attenuation curves $L_{\tau} / x_{a}$ are plotted in Figs. 9 and 10 for both values of $\delta$. Comparison of the curves shows that the structural factor $\delta$ is not a very significant parameter.

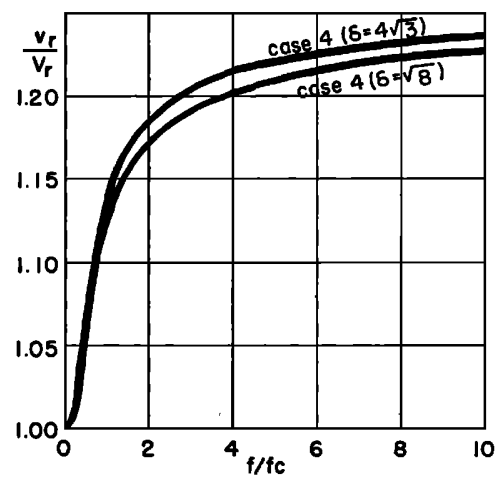

Fig. 9. Phase velocity $\nu_{r}$ of rotational waves for two values of the structural factor $\delta$.

\section{PROpagation of dilatational waVes}

Propagation of dilatational waves in the low-frequency range are governed by Eqs. (7.1) of reference 1 . To extend them to the full-frequency range we proceed as for rotational waves by multiplying the resistance coefficient by the frequency correction factor $F(\kappa)$. These equations become

$$
\begin{aligned}
& \nabla^{2}(P e+Q \epsilon)=\frac{\partial^{2}}{\partial t^{2}}\left(\rho_{11} e+\rho_{12} \epsilon\right)+b F(\kappa) \frac{\partial}{\partial t}(e-\epsilon) \\
& \nabla^{2}(Q e+R \epsilon)=\frac{\partial^{2}}{\partial t^{2}}\left(\rho_{12} e+\rho_{22} \epsilon\right)-b F(\kappa) \frac{\partial}{\partial t}(e-\epsilon) .
\end{aligned}
$$

In these equations $P Q R$ represent elastic coefficients $e$ and $\epsilon$ are the divergence of the solid and fluid displacements. A solution of the type

$$
\begin{aligned}
& e=C_{1} \exp [i(l x+\alpha t)] \\
& \epsilon=C_{2} \exp [i(l x+\alpha t)]
\end{aligned}
$$

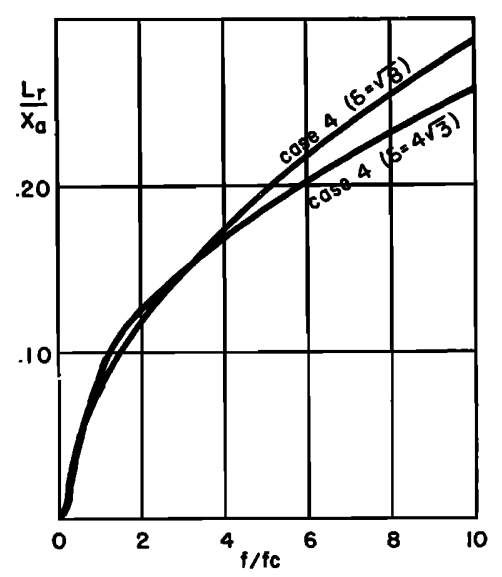

Fig. 10. Attenuation coefficient of rotational waves for two values of the structural factor $\delta$.

leads to a characteristic equation for $l^{2} / \alpha^{2}$ which may be written

$\left(\sigma_{11} \sigma_{22}-\sigma_{12}^{2}\right) z^{2}-\left(\sigma_{22} \gamma_{11}+\sigma_{11} \gamma_{23}-2 \sigma_{12} \gamma_{12}\right) z$

$$
+\left(\gamma_{11} \gamma_{22}-\gamma_{12}\right)^{2}+\frac{i b}{\alpha \rho} F(\kappa)(z-1)=0,
$$

where

$$
\sigma_{11}=\frac{P}{H} \quad \sigma_{22}=\frac{R}{H} \quad \sigma_{12}=\frac{Q}{H}
$$

$$
\begin{gathered}
H=P+R+2 Q \\
z=\frac{l^{2}}{\alpha^{2}} V_{c}^{2} \\
V_{c}^{2}=\frac{H}{\rho} .
\end{gathered}
$$


The velocity $V_{c}$ already introduced in reference 1 represents the velocity of a dilatational wave when the relative motion between fluid and solid is prevented. If we put $b=0$, i.e., in the absence of friction, Eq. (6.3) has two positive roots

$$
z_{1}=V_{c}^{2} / V_{1}^{2} \quad z_{2}=V_{c} / V_{2}^{2},
$$

where $V_{1}$ and $V_{2}$ are the velocities of the purely elastic waves. Equation (6.3) may also be written

with

$$
\left(z-z_{1}\right)\left(z-z_{2}\right)+i M(z-1)=0,
$$

$$
M=\frac{\epsilon_{1}+i \epsilon_{2}}{\sigma_{11} \sigma_{22}-\sigma_{12}{ }^{2}} .
$$

The quantities $\epsilon_{1}$ and $\epsilon_{2}$ are functions of the frequency ratio $f / f_{c}$ and $\left(\gamma_{12}+\gamma_{22}\right)$ as defined by Eqs. (5.8) and (5.9).

In the numerical discussion which follows curves have been plotted as function of $f / f_{c}$ for values of $\sigma_{i j}$ and $\gamma_{i j}$ given in Table I, and for a value $\delta=\sqrt{ } 8$ of the structural parameter.

We now compute the complex $z$-roots of Eq. (6.5) and denote the roots by $z_{I}$ and $z_{I r}$. We then evaluate

$$
\begin{aligned}
\sqrt{ } z_{I} & =R_{I}+\tau_{I} i \\
\sqrt{ } z_{I I} & =R_{I I}+\tau_{I I} i .
\end{aligned}
$$

One of the roots tends to unity for $M=\infty$, the other tends to infinity. We denote by $z_{I}$ the root which tends to unity. The phase velocities are given by

$$
\begin{aligned}
& \frac{v_{I}}{V_{c}}=\frac{1}{\left|R_{I}\right|} \\
& \frac{v_{I I}}{V_{c}}=\frac{1}{\left|\Omega_{I I}\right|} .
\end{aligned}
$$

These quantities may be plotted as function of $f / f_{c}$. The velocity $v_{I}$ tends to $V_{c}$ for zero frequency and corresponds to a wave of the first kind. The velocity $v_{I I}$ goes to zero as $\sqrt{ } f$ and corresponds to a wave of the second kind. The behavior of the waves in the vicinity of zero frequency was discussed in detail in reference 1 . The wave of the first kind is one in which the fluid and the solid tend to move in phase. The dispersion of these waves in the vicinity of zero frequency is small. The wave of the second kind is one for which the solid and the fluid tend to move in opposite phase. In the vicinity of zero frequency it behaves like a diffusion process.

If we consider now the case of large frequency the roots $z_{I}$ and $z_{I I}$ tend towards

$$
\begin{aligned}
z_{I} & =z_{1} \\
z_{I I} & =z_{2} .
\end{aligned}
$$

Hence, the phase velocities $v_{I}$ and $v_{I I}$ tend toward the velocities found in case the fluid has no viscosity,
Fig. 11. Phase velocity $\boldsymbol{v}_{I}$ of dilatational waves of the first kind.

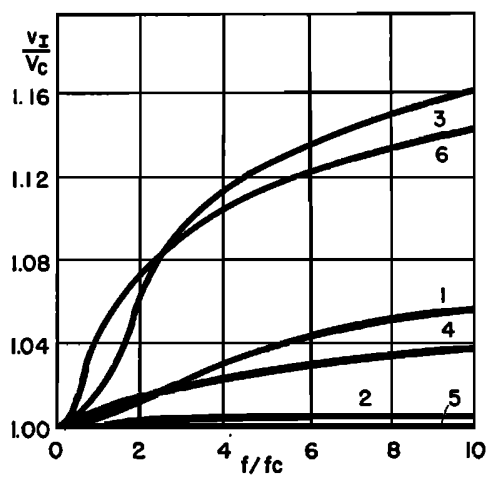

namely,

$$
\begin{gathered}
v_{I}=V_{1} \\
v_{I I}=V_{2} .
\end{gathered}
$$

The nondimensional phase velocities $v_{I} / V_{c}$ and $v_{I I} / V_{c}$ for the waves of the first and second kind are plotted in Figs. 11 and 12, for the six cases of Table I.

We now call our attention to the attenuation of these waves. Again we introduce distances $x_{I}$ and $x_{I I}$ for which the wave amplitude is multiplied by $1 / e$. With a characteristic distance defined as,

$$
L_{c}=\frac{V_{c}}{2 \pi f_{c}},
$$

the attenuation of each wave is given by

$$
\begin{aligned}
& \frac{L_{c}}{x_{I}}=\left|\tau_{I}\right| \frac{f}{f_{c}} \\
& \frac{L_{c}}{x_{I I}}=\left|\tau_{I I}\right| \frac{f}{f_{c}} .
\end{aligned}
$$

These expressions are plotted in Figs. 13 and 14 as function of $f / f_{c}$ for the six cases of Table I. We have already investigated the behavior of these curves at small frequencies in the previous paper. It was found that for the waves of the first kind the attenuation factor varies like $f^{2}$ while for the waves of the second kind it varies like $\sqrt{ } f$.

Fig. 12. Phase velocity $v_{I I}$ of dilatational waves of the second kind.

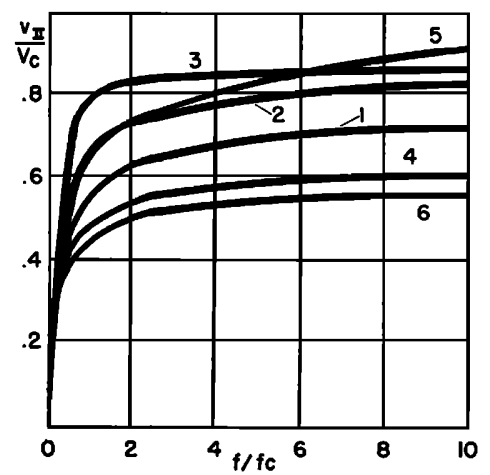




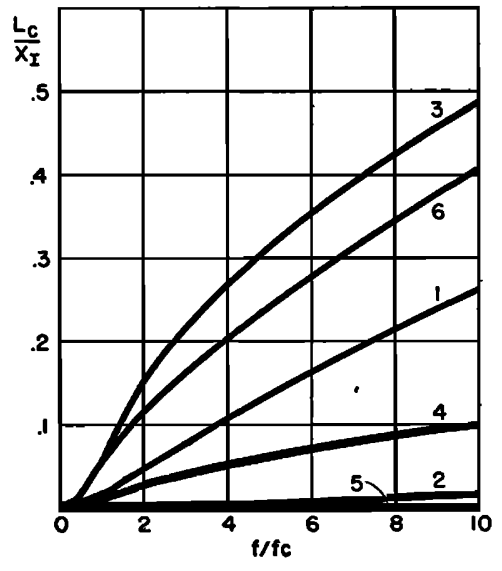

Let us now investigate the asymptotic value of the attenuation for large $f$. This amounts to calculating the roots of Eq. (6.5) for small values of $M$. We find

$$
\begin{aligned}
\left(z_{I}\right)^{\frac{3}{2}} & =\left(z_{1}\right)^{\frac{1}{2}}-\frac{i M}{2\left(z_{1}\right)^{\frac{1}{2}}} \frac{z_{1}-1}{z_{1}-z_{2}} \\
\left(z_{I I}\right)^{\frac{1}{2}} & =\left(z_{2}\right)^{\frac{1}{2}}+\frac{i M}{2\left(z_{2}\right)^{\frac{1}{2}}} \frac{z_{2}-1}{z_{1}-z_{2}} .
\end{aligned}
$$

We derive the expression for $\Omega_{I}$ and $Q_{I I}$ with the asymptotic approximations

$$
F_{r}=F_{i}=\frac{1}{2}\left(\frac{f}{f_{c}}\right)^{\frac{1}{2}} .
$$

We find the attenuations for large $f$,

$$
\begin{aligned}
& \frac{L_{c}}{x_{I}}=\frac{\left(\gamma_{12}+\gamma_{22}\right)\left(1-z_{1}\right)}{4\left(\sigma_{11} \sigma_{22}-\sigma_{12}{ }^{2}\right)\left(z_{1}\right)^{\frac{1}{2}}\left(z_{2}-z_{1}\right)}\left(\frac{f}{f_{c}}\right)^{\frac{1}{2}} \\
& \frac{L_{c}}{x_{I I}}=\frac{\left(\gamma_{12}+\gamma_{22}\right)\left(z_{2}-1\right)}{4\left(\sigma_{11} \sigma_{22}-\sigma_{12}{ }^{2}\right)\left(z_{2}\right)^{\frac{1}{2}}\left(z_{2}-z_{1}\right)}\left(\frac{f}{f_{c}}\right)^{\frac{1}{2}} .
\end{aligned}
$$

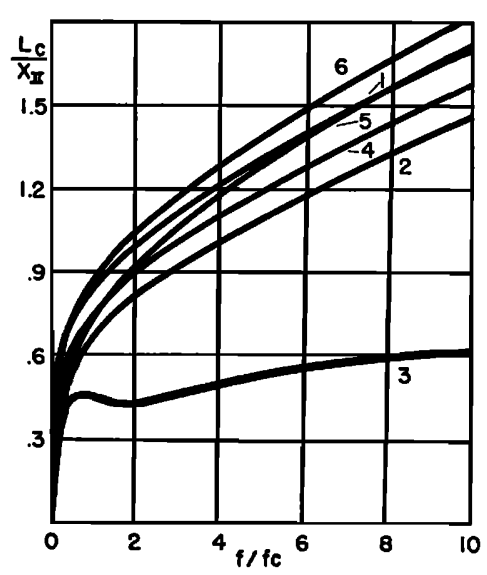

FIG. 14. Attenuation coefficient of dilatational waves of the second kind.

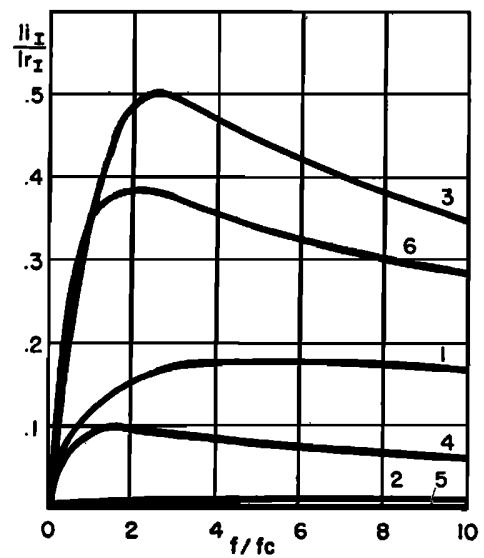

Fig. 15. Attenuation per cycle of dilatational waves of the first kind.

We see that the attenuations increase like $\sqrt{ } f$ at large frequency.

As in the case of the rotational waves we also evaluate the attenuation per cycle. We have

$$
\begin{aligned}
& \left(z_{I}\right)^{\frac{1}{2}}=\frac{V_{c}}{\alpha}\left(l_{r I}+l_{i I}\right)=R_{I}+\tau_{I} i \\
& \left(z_{I I}\right)^{\frac{1}{2}}=\frac{V_{c}}{\alpha}\left(l_{r I I}+l_{i I I}\right)=\Omega_{I I}+\tau_{I I}^{\prime} i .
\end{aligned}
$$

The attenuation per cycle for each wave is

$$
\begin{aligned}
& 2 \pi \frac{\left|l_{i I}\right|}{\left|l_{r I}\right|}=2 \pi \frac{\left|\tau_{I}\right|}{\left|\Omega_{I}\right|} \\
& 2 \pi \frac{\left|l_{i I I}\right|}{\left|l_{r I I}\right|}=2 \pi \frac{\left|\tau_{I I}\right|}{\left|\Omega_{I I}\right|}
\end{aligned}
$$

for the waves of the first and second kind, respectively. These values are plotted as functions of $f / f_{c}$ (with $\delta=\sqrt{ } 8$ ) for the six cases of Table $I$ in Figs. 15 and 16. In the vicinity of $f=0$, putting $\zeta_{1}=z_{1}-1, \zeta_{2}=z_{2}-1$,

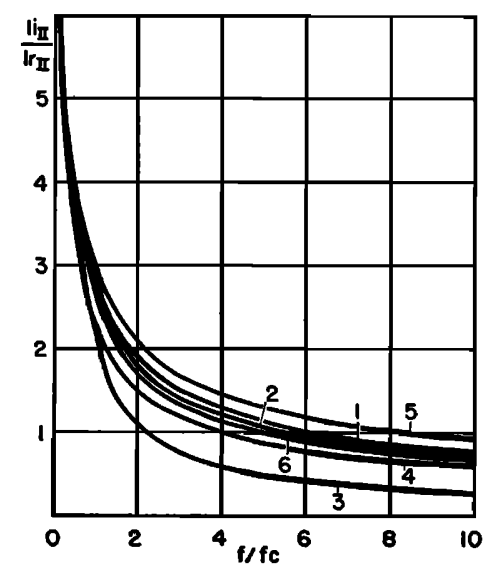

Fig. 16. Attenuation per cycle of dilatational waves of the second kind. 
FIG. 17. Group velocity $v_{g I}$ of dilatational waves of the first kind.

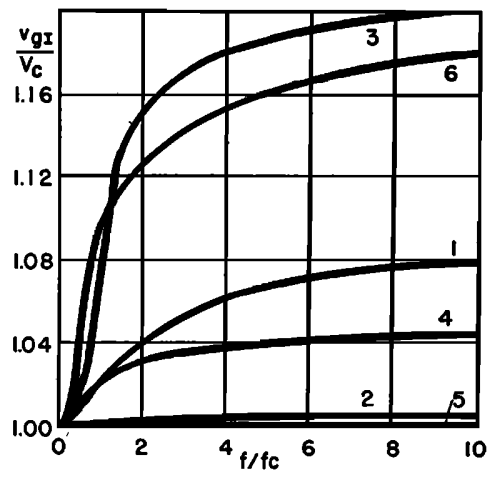

we have for the wave of the first kind:

$$
\left(z_{I}\right)^{\frac{1}{2}}=\mathcal{R}_{I}+\tau_{I} i=1+\frac{1}{2} \zeta_{1} \zeta_{2} \frac{i}{M}
$$

with

$$
\left(\frac{1}{M}\right)_{f \rightarrow 0}=\frac{\sigma_{11} \sigma_{22}-\sigma_{12}^{2}}{\gamma_{12}+\gamma_{22}} \frac{f}{f_{c}}
$$

and for the wave of the second kind:

$$
\left(z_{I I}\right)^{\frac{3}{3}}=\Re_{I I}+\tau_{I I} i=(-i)^{\frac{1}{2}}\left(\frac{\gamma_{12}+\gamma_{22}}{\sigma_{11} \sigma_{22}-\sigma_{12}{ }^{2}} \frac{f}{f_{c}}\right)^{\frac{3}{3}} .
$$

The attenuation per cycle near $f=0$ is for the waves of the first and second kind, respectively.

$$
\begin{aligned}
2 \pi \frac{\left|l_{i I}\right|}{\left|l_{r I}\right|} & =\pi \zeta_{1} \zeta_{2} \frac{\left(\sigma_{11} \sigma_{22}-\sigma_{12}{ }^{2}\right)}{\gamma_{12}+\gamma_{22}} \frac{f}{f_{c}} \\
2 \pi \frac{\left|l_{i I I}\right|}{\left|l_{r I I}\right|} & =2 \pi .
\end{aligned}
$$

The first one vanishes as $f$ near $f=0$ and the second goes to a constant $2 \pi$. The latter case as we have seen corresponds to diffusion waves. Asymptotic values of the attenuation per cycle at large frequencies are derived from the asymptotic expressions (6.13). For
FIG. 18. Group velocity $v_{g I I}$ of dilatational waves of the second kind.

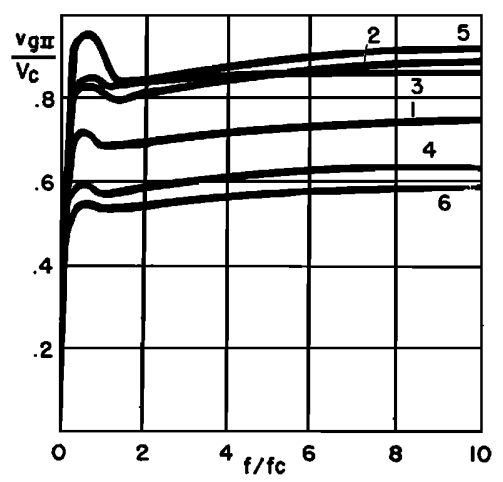

$\left(z_{I}\right)^{\frac{1}{2}}$ and $\left(z_{I I}\right)^{\frac{1}{2}}$, we find

$$
\begin{aligned}
2 \pi \frac{\left|l_{i I}\right|}{\left|l_{r I}\right|} & =\frac{\pi}{2} \frac{\left(\gamma_{12}+\gamma_{22}\right)\left(1-z_{1}\right)}{2}\left(\frac{f_{c}}{f}\right)^{\frac{1}{3}} \\
2 \pi \frac{\left.\mid \sigma_{11} \sigma_{22}-\sigma_{12}{ }^{2}\right) z_{1}\left(z_{2}-z_{1}\right)}{\left|l_{r I I}\right|} & =\frac{\pi}{2} \frac{\left(\gamma_{12}+\gamma_{22}\right)\left(z_{2}-1\right)}{2\left(\sigma_{11} \sigma_{22}-\sigma_{12}{ }^{2}\right) z_{2}\left(z_{2}-z_{1}\right)}\left(\frac{f_{c}}{f}\right)^{\frac{3}{3}} .
\end{aligned}
$$

We conclude that for waves of both kinds the attenuation per cycle vanishes as $1 / \sqrt{ } f$. The attenuation per cycle for the waves of the first kind, therefore, goes through a maximum as seen also from Fig. 15.

It remains to evaluate the group velocity of the two dilatational waves. Proceeding as for the rotational waves in the previous section, we write for the group velocity $v_{\theta I}$ and $v_{\theta I I}$ of the waves of first and second kind

$$
\begin{aligned}
& \frac{V_{c}}{v_{g I}}=\frac{d}{d\left(\frac{f}{f_{c}}\right)}\left[\frac{f}{f_{c}} \frac{V_{c}}{v_{I}}\right] \\
& \frac{V_{c}}{v_{g I I}}=\frac{d}{d\left(\frac{f}{f_{c}}\right)}\left[\frac{f}{f_{c}} \frac{V_{c}}{v_{I I}}\right] .
\end{aligned}
$$

The nondimensional group velocity variables $v_{g I} / V_{c}$ and $v_{o I I} / V_{c}$ are plotted in Figs. 17 and 18 for the six cases of Table I. 